\title{
Homogeneity of isosceles orthogonality and related inequalities
}

\author{
Cuixia $\mathrm{HaO}^{{ }^{*}}$ and Senlin $\mathrm{Wu}^{2}$
}

\author{
* Correspondence: haocuixia@hlju. \\ edu.cn \\ 'Department of Mathematics, \\ Heilongjiang University, Harbin, \\ 150080, China \\ Full list of author information is \\ available at the end of the article
}

\begin{abstract}
We study the homogeneity of isosceles orthogonality, which is one of the most important orthogonality types in normed linear spaces, from two viewpoints. On the one hand, we study the relation between homogeneous direction of isosceles orthogonality and other notions including isometric reflection vectors and $L_{2}$-summand vectors and show that a Banach space $X$ is a Hilbert space if and only if the relative interior of the set of homogeneous directions of isosceles orthogonality in the unit sphere of $X$ is not empty. On the other hand, we introduce a geometric constant $\mathrm{NH}_{X}$ to measure the non-homogeneity of isosceles orthogonality. It is proved that $0 \leq N H_{X} \leq 2$, $N H_{X}=0$ if and only if $X$ is a Hilbert space, and $N H_{X}=2$ if and only if $X$ is not uniformly non-square.

Mathematics Subject Classification (2010):

46B20; 46C15
\end{abstract}

Keywords: Birkhoff orthogonality, homogeneity of isosceles orthogonality, Roberts orthogonality, uniformly non-square

\section{Introduction}

We denote by $X$ a real Banach space with origin $o$ and norm $\|\cdot\|$, by $B_{X}$ and $S_{X}$ the unit ball and unit sphere of $X$, respectively. When the dimension of $X$ is two, $B_{X}$ and $S_{X}$ are called the unit disc and unit circle of $X$, respectively. For two linearly independent points $x$ and $y$ in $X$, we denote by $X_{x, y}$ the two-dimensional subspace of $X$ spanned by $x$ and $y$.

In a certain sense, we can say that it is the missing of an orthogonality type with "nice property" that makes non-Hilbertian Banach spaces different from Hilbert spaces (cf. characterizations of inner product spaces related to orthogonality types listed in [1], and the surveys [2] and [3]). Due to this situation, many generalized orthogonality types have been introduced into Banach spaces to act as substitutions of the orthogonality induced by inner products in Hilbert spaces. Certain property (or properties) of the orthogonality induced by an inner product is (are) missing from each of these generalized orthogonality types. For example, isosceles orthogonality introduced by James in [4], the one we study in this paper, is not homogeneous, where a vector $x$ in $X$ is said to be isosceles orthogonal to a vector $y$ in $X$ if the equality $\|x+y\|=\|x-y\|$ holds (we write $x \perp_{I} y$ for this situation). James [4] proved that $X$ is a Hilbert space if and only if isosceles orthogonality is homogeneous, i.e., if and only if the implication $x \perp_{I} y \Rightarrow x \perp_{I} \alpha y$ holds for each real number $\alpha$. For the situation of Birkhoff orthogonality (cf. [5] and [6]), where a

(c) 2011 Hao and Wu; licensee Springer. This is an Open Access article distributed under the terms of the Creative Commons Attribution License (http://creativecommons.org/licenses/by/2.0), which permits unrestricted use, distribution, and reproduction in any medium, provided the original work is properly cited. 
vector $x$ is said to be Birkhoff orthogonal to $y$ (denoted by $x \perp_{B} y$ ) if the inequality $\| x+$ $\alpha y\|\geq\| x \|$ holds for each real number $\alpha$, we know that this orthogonality is not symmetric, i.e., $x \perp_{B} y$ does not necessarily imply that $y \perp_{B} x$. We also need the notion of Roberts orthogonality. A vector $x$ is said to be Roberts orthogonal to another vector $y$ (denoted by $x \perp_{R} y$ ) if the equality $\|x+\alpha y\|=\|x-\alpha y\|$ holds for each real number $\alpha$ (cf. [7]). Roberts orthogonality implies both Birkhoff orthogonality and isosceles orthogonality. More precisely, the implications $x \perp_{R} y \Rightarrow x \perp_{B} y$ and $x \perp_{R} y \Rightarrow x \perp_{I} y$ hold. Roberts orthogonality is both homogeneous and symmetric, but it does not have the existence property (cf. Example 2.1 in [4]): there exists a Minkowski plane (i.e., a real two-dimensional Banach space) such that

$$
x \perp_{R} y \Rightarrow\|x\| \cdot\|y\|=0 .
$$

Although isosceles orthogonality is not homogeneous in general, it is possible that, for a Banach space that is not a Hilbert space, there exists a vector $x \in S_{X}$ such that the implication

$$
\forall y \in X, x \perp_{I} y \Rightarrow x \perp_{R} y
$$

holds. Such a unit vector $x$ is said to be a homogeneous direction of isosceles orthogonality. In the following, we denote by $H_{X}$ the set of all homogeneous directions of isosceles orthogonality in $X$. In Section 2, we study the relation of homogeneous direction of isosceles orthogonality to other notions including isometric reflection vectors and $L_{2}$-summand vectors (see Section 2 for the definitions) and prove a new characterization of Hilbert spaces.

In the meantime, we provide a quantitative characterization of the non-homogeneity of isosceles orthogonality by introducing a new geometric constant $N H_{X}$. We show that $N H_{X}=0$ if and only if isosceles orthogonality is homogeneous and $N H_{X}=2$ if and only if the underlying space is not uniformly non-square.

\section{Homogeneous directions of isosceles orthogonality}

First, we study the relation of homogeneous directions of isosceles orthogonality to other notions.

\subsection{Relations to isometric reflection vectors and $L_{2}$-summand vectors}

A reflection on $X$ is an operator defined as follows: $T_{x, x^{*}}: z \rightarrow z-2 x^{*}(z) \cdot x$, where $x \in$ $X, x^{*} \in X^{*}$, and $x^{*}(x)=1$. Let $x$ be a point in $S_{X}$. If there exists a point $x^{*} \in S_{X^{*}}$ such that the reflection $T_{x, x^{*}}$ is an isometry then $x$ is said to be an isometric reflection vector and $x^{*}$ is said to be the corresponding isometric reflection functional. For any isometric reflection vector $x$, there is a unique isometric reflection functional $x$ corresponding to it (cf. [8]). For the relation between isometric reflection vectors and Roberts orthogonality, Chan $\mathrm{He}$ et al. proved the following lemma.

Lemma 1 ([9]) Let $X$ be a real Banach space, $x \in S_{X}, x^{*} \in S_{X^{*}}$ and $T_{x, x^{*}}$ be a reflection. Then, $T_{x, x^{*} i s}$ an isometric reflection if and only if

$$
x \perp_{R} H:=\left\{z: z \in X, x^{*}(z)=0\right\} .
$$

From Lemma 1, one can see that the notions of "homogeneous direction of isosceles orthogonality" and "isometric reflection vector" are closely connected. One may even 
expect that these two notions coincide. However, this is not true even when the underlying space is two-dimensional.

Example 1 Let $X=\left(\mathbb{R}^{2},\|\cdot\|_{\infty}\right), x=(0,1), y=(1,0)$, and $z=(2,1)$. Then, it is not difficult to verify that $x$ is an isometric reflection vector. However, one can observe that $x$ $\notin H_{X}$ since $x \perp_{I} z$ but $x \not \not_{I}(z / 2)$.

In the meantime, we have the following proposition.

Proposition 1 Let $X$ be a Minkowski plane and $x \in H_{X}$. Then, $x$ is an isometric reflection vector.

Proof Let $y$ be a point in $S_{X}$ such that $x \perp_{I} y$. Then, $x \perp_{R} y$ since $x \in H_{X}$. Recall that Roberts orthogonality implies Birkhoff orthogonality. Hence $x \perp_{B} y$. Thus, there exists a functional $x^{*} \in S_{X^{*}}$ such that $x^{*}(x)=1$ and $x^{*}(y)=0$ (cf. [6]). Then, $T_{x, x^{*}}$ is a reflection and the set $H:=\left\{z \in X: x^{*}(z)=0\right\}$ is precisely the line passing through $-y$ and $y$. Since Roberts orthogonality is homogeneous, we have $x \perp_{R} H$. Then, it follows from Lemma 1 that $T_{x, x^{*}}$ is an isometry and $x$ is an isometric reflection vector.

Example 1 shows that the converse of Proposition 1 is not true in general, but it holds when the underlying Minkowski plane is strictly convex. More precisely, we have the following proposition.

Proposition 2 Let $X$ be a Minkowski plane, $x \in S_{X}$ be an isometric reflection vector. If there does not exist a nontrivial line segment contained in $S_{X}$ and parallel to the line passing through $-x$ and $x$ then $x \in H_{X}$.

Proof Since $x$ is an isometric reflection vector, by Lemma 1, there exists a point $y \in$ $S_{X}$ such that $x \perp_{R}\{\alpha y: \alpha \in \mathbb{R}\}$. On the other hand, by the assumption of the proposition and Theorem 2.3 in [10] (see also [11]), for each number $\rho>0$, there exist precisely two points $p$ and $-p$ in $X$ such that $\|p\|=\rho$ and $x \perp_{I} p$ hold. Clearly, these two points have to be the points of intersection of the line $\{\alpha y: \alpha \in \mathbb{R}\}$ and the sphere $\rho S_{X}$. Thus, for each point $z \in X$ satisfying $x \perp_{I} z$, we have $z=\|z\| y$ or $z=-\|z\| y$. Since Roberts orthogonality is homogeneous, this means that $x \perp_{R} z$. Thus, $x \in H_{X}$.

Proposition 1 does not hold in higher dimensional cases. See the following example.

Example 2 Let $X=\left(\mathbb{R}^{3},\|\cdot\|_{\infty}\right)$ and $x=(1,1,1)$. Then, $x \in H_{X}$, and it is not an isometric reflection vector.

Proof Let $y$ be an arbitrary point in $S_{X}$ such that $x \perp_{I} y$. Then, it is clear that $x$ and $y$ are linearly independent. Next, we show that $x \perp_{R} y$.

Assume that $y=(\alpha, \beta, \gamma)$. Then, since $y \in S_{X}, \max \{|\alpha|,|\beta|,|\gamma|\}=1$. We only deal with the case when $|\alpha|=1$, and the other two cases can be proved in a similar way.

By replacing $y$ with $-y$ if it is necessary, we may assume that $\alpha=1$. Then,

$$
\|x+y\|=\max \{2,|1+\beta|,|1+\gamma|\}=2
$$

and

$$
|| x-y||=\max \{|1-\beta|,|1+\gamma|\} .
$$

From the fact that $x \perp_{I} y$ it follows that

$$
|1-\beta|=2 \text { or }|1-\gamma|=2 .
$$

We only need to consider the subcase when $\beta=-1$, and the other subcase when $\gamma=$ -1 can be proved similarly. 
For any real number $\mu$, we have the following equations:

$$
|| x+\mu y||=\max \{|1+\mu|,|1-\mu|,|1+\mu \gamma|\}
$$

and

$$
|| x-\mu \gamma||=\max \{|1-\mu|,|1+\mu|,|1-\mu \gamma|\} .
$$

Since

$$
|1+\mu \gamma|,|1-\mu \gamma| \leq \max \{|1+\mu|,|1-\mu|\}
$$

we have that

$$
\|x+\mu y\|=\|x-\mu y\|,
$$

which implies that $x \perp_{R} y$.

In the rest of the proof, we show that $x$ is not an isometric reflection vector.

Otherwise, there exists a hyperplane $H$ passing through the origin $o$ such that $x \perp_{R}$ $H$. Let $z_{1}=(-1,-1,1)$ and $z_{2}=(-1,1,-1)$. Then, it is clear that $x \perp_{R} z_{1}$ and $x \perp_{R} z_{2}$. Since $z_{1}$ is the unique (except for the sign) point in $S_{X_{x, z_{1}}}$ such that $x \perp_{R} z_{1}$, and $H$ intersects $X_{x, z_{1}}$, we have that $z_{1} \in H$. Similarly, $z_{2} \in H$. However, for the point $w=$ $\left(z_{1}+z_{2}\right) / 2$, we have

$$
\|x+w\|=1,\|x-w\|=2,
$$

which imply that $x \not_{R} w$. This is a contradiction to the fact that $H$ is a hyperplane in $X$.

Nevertheless, we have the following lemma.

Lemma 2 If $x \in H_{X}$ is a smooth point of $S_{X}$ then $x$ is an isometric reflection vector, and therefore, $x$ is Roberts orthogonal to a hyperplane.

Proof By Lemma 1, it suffices to show that $x$ is Roberts orthogonal to a hyperplane. Since $x$ is a smooth point, there exists a unique hyperplane $H$ such that $x \perp_{B} H$. In the following, we show that $x \perp_{R} H$.

For each vector $z \in H \backslash\{0\}$, there exists a unit vector $z^{\prime} \in X_{x, z}$ such that $x \perp_{I} z^{\prime}$. From the relation $x \in H_{X}$, it follows that $x \perp_{R} z^{\prime}$, which implies $x \perp_{B} z^{\prime}$. Since $x$ is a smooth point, either $z /\|z\|=z^{\prime}$ or $z /\|z\|=-z^{\prime}$ holds. Thus, $x \perp_{R} z$. The case when $z=o$ is trivial.

Let $M$ be a closed subspace of $X$. If there exists another closed subspace $N$ of $X$ such that $X=M \oplus N$ and that, for each pair of points $m \in M$ and $n \in N$, the equality

$$
\|m+n\|^{2}=\|m\|^{2}+\|n\|^{2}
$$

holds, then $M$ is said to be an $L_{2}$-summand subspace (cf. [12]). Note that, when $M$ is an $L_{2}$-summand subspace, $N$ is uniquely determined. Let $x$ be a point in $X$. If the subspace spanned by $x$ is an $L_{2}$-summand subspace then $x$ is said to be an $L_{2}$-summand vector.

Theorem 1 Let $x \in S_{X}$ be an $L_{2}$-summand vector. Then, $x \in H_{X}$.

Proof We denote by $M$ the one-dimensional subspace spanned by $x$, by $N$ the closed subspace of $X$ such that $X=M \oplus N$ and that the equality

$$
\|m+n\|^{2}=\left.\|m\|\right|^{2}+\|n\|^{2}
$$


holds for each pair of points $m \in M$ and $n \in N$. Let $y$ be an arbitrary point in $X$ such that $x \perp_{I} y, y_{M} \in M$, and $y_{N} \in N$ be the two points such that $y=y_{M}+y_{N}$. Then,

$$
\|x+y\|=\sqrt{\|x+y\|^{2}}=\sqrt{\left\|x+y_{M}+y_{N}\right\|^{2}}=\sqrt{\left\|x+y_{M}\right\|^{2}+\left\|y_{N}\right\|^{2}}
$$

and

$$
\|x-y\|=\sqrt{\|x-y\|^{2}}=\sqrt{\left\|x-y_{M}-y_{N}\right\|^{2}}=\sqrt{\left\|x-y_{M}\right\|^{2}+\left\|y_{N}\right\|^{2}} .
$$

It follows that

$$
\left\|x+y_{M}\right\|=\left\|x-y_{M}\right\| \text {. }
$$

This equation holds only if $y_{M}=o$. Thus, we have that $y=y_{N} \in N$. Next, we show that, for each point $z \in N, x \perp_{I} z$. Actually, this is an easy consequence of the equations

$$
\|x+z\|=\sqrt{\|x+z\|^{2}}=\sqrt{\|x\|^{2}+\|z\|^{2}}
$$

and

$$
\|x-z\|=\sqrt{\|x-z\|^{2}}=\sqrt{\|x\|^{2}+\|z\|^{2}} .
$$

Since $N$ is a linear subspace of $X$, it follows that $x \perp_{R} z$ holds for each point $z \in N$. We have shown that, for each point $y \in X, x \perp_{I} y$ implies that $x \perp_{R} y$, i.e., $x \in H_{X}$.

\subsection{A characterization of Hilbert spaces}

Theorem 2 Let $X$ be a Banach space with $\operatorname{dim} X \geq 2$. Then, $X$ is a Hilbert space if and only if the relative interior of $H_{X}$ in $S_{X}$ is not empty.

Proof Clearly, if $X$ is a Hilbert space then isosceles orthogonality coincides with Roberts orthogonality, which implies that $H_{X}=S_{X}$.

Now assume that the relative interior of $H_{X}$ in $S_{X}$, which is denoted by $P$, is not empty. By Theorem 2.2 in [8], it suffices to show that each point $x$ in $P$ is an isometric reflection vector. By Lemma 2, we only need to show that each point $x$ in $P$ is a smooth point.

Let $x$ be an arbitrary point in $P$. Suppose to the contrary that $x$ is not a smooth point. Then, there exists a two-dimensional subspace $Y$ containing $x$ such that $x$ is not a smooth point of $S_{Y}$. Let $w$ be a point in $S_{Y}$ such that $x \perp_{B} w$. Since $x$ is a relative interior point of $P$, it is also a relative interior point of $P \cap S_{Y}$. Thus, there exist two points $u$ and $v$ in $S_{Y}$ such that $x$ is a relative interior point (with respect to $S_{Y}$ ) of the set

$$
\operatorname{arc}(u, v):=\{\lambda u+\mu v: \lambda, \mu \geq 0\} \cap S_{Y} \subset P .
$$

Moreover, we may assume, without loss of generality, that $v \in \operatorname{arc}(x, w)$ and that each point of $\operatorname{arc}(u, v) \backslash\{x\}$ is a smooth point. The points $u$ and $v$ are also chosen in a way such that there exist two numbers $\alpha_{0} \geq 0, \beta_{0} \leq 0$ and that the relations $u \perp_{B}\left(\alpha_{0} x\right.$ $+w)$ and $v \perp_{B}\left(\beta_{0} x+w\right)$ hold. I.e., we assume that the supporting lines of $B_{Y}$ at $u$ and $v$ both intersect the line passing through $w$ and parallel to $\langle-x, x\rangle$. Let $\left\{u_{n}\right\}$ and $\left\{v_{n}\right\}$ be two sequences such that

$$
\begin{aligned}
& \left\{u_{n}\right\} \subset \operatorname{arc}(u, x):=\{\lambda u+\mu x: \lambda, \mu>0\} \cap S_{Y}, \\
& \left\{v_{n}\right\} \subset \operatorname{arc}(v, x):=\{\lambda v+\mu x: \lambda, \mu>0\} \cap S_{Y},
\end{aligned}
$$


and

$$
\lim _{n \rightarrow \infty} u_{n}=\lim _{n \rightarrow \infty} v_{n}=x
$$

Then, there exist two sequences of numbers $\left\{\alpha_{n}\right\}$ and $\left\{\beta_{n}\right\}$ such that

$$
u_{n} \perp_{B}\left(\alpha_{n} x+w\right) \text { and } v_{n} \perp_{B}\left(\beta_{n} x+w\right) .
$$

By extracting two subsequences if it is necessary, we may assume, without loss of generality, that there exist two numbers $A$ and $B$ such that

$$
\lim _{n \rightarrow \infty} \alpha_{n}=B \text { and } \lim _{n \rightarrow \infty} \beta_{n}=A .
$$

Then, since $S_{Y}$ is a closed convex curve, $B \geq A$. Now, we have that

$$
x \perp_{B}(B x+w) \text { and } x \perp_{B}(A x+w) .
$$

Since $x$ is not a smooth point, $B>A$. Recall that Roberts orthogonality implies Birkhoff orthogonality. Thus,

$$
u_{n} \perp_{R}\left(\alpha_{n} x+w\right) \text { and } v_{n} \perp_{R}\left(\beta_{n} x+w\right) \text {. }
$$

This implies that

$$
u_{n} \perp_{I}\left(\alpha_{n} x+w\right) \text { and } v_{n} \perp_{I}\left(\beta_{n} x+w\right) \text {. }
$$

Thus,

$$
x \perp_{I}(B x+w) \text { and } x \perp_{I}(A x+w) .
$$

Since $x \in H_{X}$, we have

$$
x \perp_{I}\left(\frac{B x+w}{\|B x+w\|}\right) \text { and } x \perp_{I}\left(\frac{A x+w}{\|A x+w\|}\right) .
$$

Due to the uniqueness property of isosceles orthogonality on the unit sphere, this is impossible. It follows that $x$ is a smooth point.

\section{A measure of non-homogeneity of isosceles orthogonality}

In this section, we introduce the following measures of non-homogeneity of isosceles orthogonality: the constant

$$
N H_{X}=\sup _{\alpha>0}\left\{\frac{\|x+\alpha y\|-\|x-\alpha y\|}{\alpha}: x, y \in S_{X}, x \perp_{I} y\right\}
$$

and its local version for $x \in S_{X}$

$$
N H_{X}(x)=\sup _{\alpha>0}\left\{\frac{\|x+\alpha y\|-\|x-\alpha y\|}{\alpha}: y \in S_{X}, x \perp_{I} y\right\} .
$$

Now, the following result follows from Theorem 2 and the observation that $x \in H_{X}$ if and only if $N H_{X}(x)=0$.

Theorem 3 Let $X$ be a Banach space with $\operatorname{dim} X \geq 2$. If the relative interior of $\left\{x \in S_{X}\right.$ $\left.: N H_{X}(x)=0\right\}$ in $S_{X}$ is not empty then $X$ is a Hilbert space.

For the discussion in this section, we need to introduce the so called non-square constant (or, James constant. Cf. [13] and [14]; see also [15] for a generalized version): 


$$
\begin{aligned}
J(X) & :=\sup \left\{\min \{\|x+y\|,\|x-y\|\}: x, y \in S_{X}\right\} \\
& =\sup \left\{\|x-y\|: x, y \in S_{X}, x \perp_{I} y\right\} .
\end{aligned}
$$

For any Banach space $X$, we have $\sqrt{2} \leq J(X) \leq 2$. It is well known that a Banach space $X$ is uniformly non-square if and only if $J(X)<2$. Some preliminaries about ultrapower are also necessary. Let $\mathcal{U}$ be an ultrafilter on $\mathbb{N}$. A sequence $\left\{x_{n}\right\}$ in $X$ converges to $x$ with respect to $\mathcal{U}$, denoted by $\lim _{\mathcal{U}} x_{n}=x$, if, for each neighborhood $U$ of $x$, $\left\{i \in \mathbb{N}: x_{i} \in U\right\} \in \mathcal{U}$. The ultrapower of $X$, which is denoted by $\tilde{X}$, is the quotient space $l_{\infty}(X) / N_{\mathcal{U}}(X)$ equipped with the quotient norm, where

$$
\begin{gathered}
\left.l_{\infty}(X)=\left\{\left(x_{n}\right) \subset X:\left\|\left(x_{n}\right)\right\|=\sup _{n \in \mathbb{N}}\left\|x_{n}\right\|<\infty\right\}\right\}, \\
N_{\mathcal{U}}(X)=\left\{\left(x_{n}\right) \in l_{\infty}(X): \lim _{\mathcal{U}}\left\|x_{n}\right\|=0\right\},
\end{gathered}
$$

and $\|\tilde{x}\|=\lim _{\mathcal{U}}\left\|x_{n}\right\|$ for $\tilde{x}=\left(x_{n}\right)_{\mathcal{U}} \in \tilde{X}$. For more information about ultra-techniques in Banach space theory, we refer to [16] and [17].

First, we prove the following inequality between $N H_{X}$ and $J(X)$.

Lemma 3 Let $X$ be a Banach space with $\operatorname{dim} X \geq 2$. Then,

$$
N H_{X} \leq 2(J(X)-1) .
$$

Proof Let $x$ and $y$ be two arbitrary unit vectors that are isosceles orthogonal to each other and $\alpha$ be an arbitrary positive real number. Without loss of generality, we may assume that $\|x+\alpha y\| \geq\|x-\alpha y\|$. In the following, we distinguish two cases.

Case 1: $0<\alpha \leq 1$. It follows from the convexity of $f(\alpha)=\|x+\alpha y\|$ that

$$
\frac{\|x+\alpha y\|-\|x-\alpha y\|}{2 \alpha} \leq \frac{\|x+y\|-\|x\|}{1} \leq J(X)-1 .
$$

Thus

$$
\frac{\|x+\alpha y\|-\|x-\alpha y\|}{\alpha} \leq 2(J(X)-1) .
$$

Case 2: $\alpha>1$. By the triangle inequality, we have

$$
\frac{\|x+\alpha y\|-\|x-\alpha y\|}{\alpha} \leq \frac{\|x+y\|+(\alpha-1)\|y\|-\|x-y\|}{\alpha}=1-\frac{1}{\alpha} .
$$

Similarly, we have

$$
\frac{\|x+\alpha y\|-\|x-\alpha y\|}{\alpha} \leq \frac{2}{\alpha} .
$$

Thus,

$$
\frac{\|x+\alpha y\|-\|x-\alpha y\|}{\alpha} \leq \min _{\alpha>1}\left(1-\frac{1}{\alpha}, \frac{2}{\alpha}\right) \leq \frac{2}{3} \leq 2(J(X)-1) .
$$

The desired inequality now follows directly from the definitions of $N H_{X}$ and $J(X)$.

Lemma 4 Let $X$ be a Banach space with $\operatorname{dim} X \geq 2$. If $N H_{X}=2$ then $J(X)=2$.

Proof If $N H_{X}=2$ then $2=N H_{X} \leq 2(J(X)-1) \leq 2$. Thus $J(X)=2$.

For the lower and upper bounds of $N H_{X}$ and $N H_{X}(x)$, we have the following theorem. 
Theorem 4 Let $X$ be a Banach space with $\operatorname{dim} X \geq 2$. Then,

$$
0 \leq N H_{X} \leq 2 \text { and } 0 \leq N H_{X}(x) \leq 2, \forall x \in S_{X} .
$$

$N H_{X}=0$ if and only if $X$ is a Hilbert space and $N H_{X}=2$ if and only if $X$ is not uniformly non-square.

Proof To prove the inequalities $0 \leq N H_{X} \leq 2$, it suffices to show that $0 \leq N H_{X}(x) \leq 2$ hold for each $x \in S_{X}$, which follow from the following observation: for each number $\alpha>0$,

$$
\frac{\|x+\alpha y\|-\|x-\alpha y\|}{\alpha} \leq \frac{\|x+\alpha y\|-\|x-\alpha y\| \mid}{\alpha} \leq \frac{\min \{2,2 \alpha\}}{\alpha} \leq 2 .
$$

By Theorem 3, it is clear that if $N H_{X}=0$ then $X$ is a Hilbert space. Conversely, if $X$ is Hilbert space then isosceles orthogonality coincides with Roberts orthogonality, which implies that $N H_{X}=0$. In the following, we prove that $N H_{X}=2$ if and only if $J$ $(X)=2$. By Lemma 4 , we only need to show the implication $J(X)=2 \Rightarrow N H_{X}=2$.

Suppose that $J(X)=2$ holds. Then, there exist $\left\{x_{n}\right\},\left\{y_{n}\right\} \subset S_{X}$ such that $x_{n} \perp_{I} y_{n}$ holds for each $n$ and

$$
\lim _{n \rightarrow \infty}\left\|x_{n}+y_{n}\right\|=2 \text {. }
$$

Let $\tilde{x}=\left(x_{n}\right) \mathcal{U}$ and $\tilde{y}=\left(y_{n}\right) \mathcal{U}$. Then, $\tilde{x}, \tilde{y} \in S_{\tilde{X}}$ and $\|\tilde{x}+\tilde{y}\|=\|\tilde{x}-\tilde{y}\|=2$. Thus, the unit circle of the two-dimensional subspace $\tilde{X}_{\tilde{x}, \tilde{y}}$ of $\tilde{X}$ is the parallelogram with $\tilde{x}, \tilde{y}$, $-\tilde{y}$, and $-\tilde{\gamma}$ as vertices. Then, for any number $\alpha, \beta \geq 0$ satisfying $\alpha+\beta>0$, we have

$$
\|\alpha \tilde{x}+\beta \tilde{y}\|=(\alpha+\beta)\left\|\frac{\alpha}{\alpha+\beta} \tilde{x}+\frac{\beta}{\alpha+\beta} \tilde{y}\right\|=\alpha+\beta .
$$

For each sufficiently large $k \in \mathbb{N}$ and each $n \in \mathbb{N}$, let $v_{n, k}$ be a point in the unit circle of $X_{x_{n}, y_{n}}$ such that $\left|v_{n, k}-x_{n}\right| \mid=\frac{1}{k}$ and that $x_{n} \in \operatorname{arc}\left(v_{n, k}, y_{n}\right)$; let $u_{n, k}$ be a point in $\operatorname{arc}\left(x_{n}\right.$, $\left.y_{n}\right)$ such that $u_{n, k} \perp_{I} v_{n, k}$. Then, there exist $\left\{\alpha_{n, k}\right\},\left\{\beta_{n}, k\right\},\left\{\gamma_{n, k}\right\}$, and $\left\{\eta_{n}, k\right\} \subset(0,+\infty)$ such that $x_{n}=\alpha_{n, k} v_{n, k}+\beta_{n, k} y_{n}$ and $u_{n, k}=\gamma_{n, k} x_{n}+\eta_{n, k} y_{n}$. By extracting subsequences if it is necessary, we may assume that $\left\{u_{n, k}\right\},\left\{v_{n, k}\right\},\left\{\alpha_{n, k}\right\},\left\{\beta_{n, k}\right\},\left\{\gamma_{n, k}\right\}$, and $\left\{\eta_{n, k}\right\}$ all converge as $k$ tends to infinity.

Let $\tilde{u}_{k}=\left(u_{n, k}\right) \mathcal{U}$ and $\tilde{v}_{k}=\left(v_{n, k}\right) \mathcal{U}$. Then, $\tilde{u}_{k}, \tilde{v}_{k} \in S_{\tilde{X}_{\tilde{x}, \tilde{p}}} \tilde{u}_{k} \perp_{I} \tilde{v}_{k}$, and $\left\|\tilde{v}_{k}-\tilde{x}\right\|=\frac{1}{k}$. Moreover, $\tilde{x} \in \operatorname{arc}\left(\tilde{v}_{k}, \tilde{y}\right)$ and $\tilde{u}_{k} \in \operatorname{arc}(\tilde{x}, \tilde{y})$. It is not difficult to verify the following two equalities

$$
\tilde{v}_{k}=-\frac{1}{2 k} \tilde{y}+\left(1-\frac{1}{2 k}\right) \tilde{x} \text { and } \tilde{u}_{k}=\left(1-\frac{1}{2 k}\right) \tilde{y}+\frac{1}{2 k} \tilde{x} .
$$

For sufficiently large $k$ and $\alpha \leq \frac{1}{2 k}$, we have

$$
\begin{aligned}
& \frac{1}{\alpha}\left(\left\|\tilde{u}_{k}+\alpha \tilde{v}_{k}\right\|-\left\|\tilde{u}_{k}-\alpha \tilde{v}_{k}\right\|\right) \\
= & \frac{1}{\alpha}\left(\left\|\left(1-\frac{1}{2 k}-\frac{\alpha}{2 k}\right) \tilde{y}+\left(\frac{1}{2 k}+\alpha-\frac{\alpha}{2 k}\right) \tilde{x}\right\|\right. \\
& \left.-\left\|\left(1-\frac{1}{2 k}+\frac{\alpha}{2 k}\right) \tilde{\gamma}+\left(\frac{1}{2 k}-\alpha+\frac{\alpha}{2 k}\right) \tilde{x}\right\|\right) \\
= & \frac{1}{\alpha}\left(1-\frac{1}{2 k}-\frac{\alpha}{2 k}+\frac{1}{2 k}+\alpha-\frac{\alpha}{2 k}-\left(1-\frac{1}{2 k}+\frac{\alpha}{2 k}+\frac{1}{2 k}-\alpha+\frac{\alpha}{2 k}\right)\right) \\
= & 2-\frac{2}{k} .
\end{aligned}
$$


Thus,

$$
\begin{aligned}
N H_{X} & \geq \lim _{n \rightarrow \infty} \frac{1}{\alpha}\left(\left\|u_{n, k}+\alpha v_{n, k}\right\|-\left\|u_{n, k}-\alpha v_{n, k}\right\|\right) \\
& =\lim _{\mathcal{u}} \frac{1}{\alpha}\left(\left\|u_{n, k}+\alpha v_{n, k}\right\|-\left\|u_{n, k}-\alpha v_{n, k}\right\|\right) \\
& =\frac{1}{\alpha}\left(\left\|\tilde{u}_{k}+\alpha \tilde{v}_{k}\right\|-\left\|\tilde{u}_{k}-\alpha \tilde{u}_{k}\right\|\right)=2-\frac{2}{k} .
\end{aligned}
$$

Since $1 / k$ tends to 0 when $k$ tends to infinity, we have $N H_{X}=2$.

\begin{abstract}
Acknowledgements
The authors are grateful for useful advice from the anonymous referee which leads to a improvement of the proof of Theorem 4. The research of the first named author is supported by a grant from Ministry of Education of Heilongjiang Province supporting overseas returned scholars. The research of the second named author is supported by National Nature Science Foundation of China (grant number 11001068), a foundation from the Ministry of Education of Heilongjiang Province (grant number 11541069), a foundation from Harbin University of Science and Technology (grant number 2009YF028), and by the Scientific Research Foundation for the Returned Overseas Chinese Scholars, State Education Ministry.
\end{abstract}

\title{
Author details
}

'Department of Mathematics, Heilongjiang University, Harbin, 150080, China 2Department of Applied Mathematics, Harbin University of Science and Technology, Harbin, 150080, China

\section{Authors' contributions}

$\mathrm{CH}$ participated in the design and coordination of the study, and the proof of some results. SW conceived of the study, participated in the design of the proof and drafted the manuscript. All authors read and approved the final manuscript.

\section{Competing interests}

The authors declare that they have no competing interests.

Received: 14 May 2011 Accepted: 11 October 2011 Published: 11 October 2011

\section{References}

1. Amir, D: Characterizations of Inner Product Spaces, Operator Theory: Advances and Applications. Birkhäuser, Basel20 (1986)

2. Alonso, J, Benítez, C: Orthogonality in normed linear spaces: a survey. Part I. Main properties. Extracta Math. 3(1), 1-15 (1988)

3. Alonso, J, Benítez, C: Orthogonality in normed linear spaces: a survey. Part II. Relations between main orthogonalities. Extracta Math. 4(3), 121-131 (1989)

4. James, RC: Orthogonality in normed linear spaces. Duke Math J. 12, 291-302 (1945). doi:10.1215/50012-7094-45-01223-3

5. Birkhoff, G: Orthogonality in linear metric spaces. Duke Math J. 1(2), 169-172 (1935). doi:10.1215/S0012-7094-35-00115-6

6. James, RC: Orthogonality and linear functionals in normed linear spaces. Trans Am Math Soc. 61, 265-292 (1947). doi:10.1090/S0002-9947-1947-0021241-4

7. Roberts, BD: On the geometry of abstract vector spaces. Tôhoku Math J. 39, 42-59 (1934)

8. Guerrero, JB, Palacios, AR: Isometric reflections on Banach spaces after a paper of A. Skorik and M. Zaidenberg: "On isometric reflections in Banach spaces" [Mat. Fiz. Anal. Geom 4(1-2), 212-247 (1997); MR1484353 (90a:46025)]. Rocky Mountain J Math. 30(1), 63-83 (2000). doi:10.1216/rmjm/1022008976

9. He, C, Martini, H, Wu, S: On bisectors for convex distance functions. Proceedings of 2011 Eighth International Symposium on Voronoi Diagrams in Science and Engineering. 23-30 (2011)

10. Ji, D, Li, J, Wu, S: On the uniqueness of isosceles orthogonality in normed linear spaces. Results Math. 59, 157-162 (2011). doi:10.1007/s00025-010-0069-6

11. Alonso, J: Uniqueness properties of isosceles orthogonality in normed linear spaces. Ann Sci Math Québec. 18(1), 25-38 (1994)

12. Aizpuru, A, García-Pacheco, FJ: P'-summand vectors in Banach spaces. Proc Am Math Soc. 134(7), 2109-2115 (2006). doi:10.1090/S0002-9939-06-08243-8

13. Gao, J, Lau, KS: On the geometry of spheres in normed linear spaces. J Aust Math Soc (Series A). 48(01), 101-112 (1990). doi:10.1017/S1446788700035230

14. Ji, D, Zhan, D: Some equivalent representations of nonsquare constants and its applications. Northeast Math J. 15, 439-444 (1999)

15. He, C, Cui, Y: Some properties concerning Milman's moduli. J Math Anal Appl. 329(2), 1260-1272 (2007). doi:10.1016/j. jmaa.2006.07.046

16. Aksoy, AG, Khamsi, MA: Nonstandard Methods in Fixed Point Theory. Springer, New York (1990)

17. Sims, B: Ultra-Techniques in Banach Space Theory, Queen's Papers in Pure and Applied Mathematics. Queen's University, Kingston, Canada60 (1982)

doi:10.1186/1029-242X-2011-84

Cite this article as: Hao and Wu: Homogeneity of isosceles orthogonality and related inequalities. Journal of Inequalities and Applications 2011 2011:84. 